REVIEW

\title{
Advances in wound healing: topical negative pressure therapy
}

S M Jones, P E Banwell, P G Shakespeare

Postgrad Med J 2005;81:353-357. doi: 10.1136/pgmj.2004.026351

In clinical practice many wounds are slow to heal and difficult to manage. The recently introduced technique of topical negative pressure therapy (TNP) has been developed to try to overcome some of these difficulties. TNP applies a controlled negative pressure to the surface of a wound that has potential advantages for wound treatment and management. Although the concept itself, of using suction in wound management is not new, the technique of applying a negative pressure at the surface of the wound is. This paper explores the origins and proposed mechanisms of action of TNP therapy and discusses the types of wounds that are thought to benefit most from use of this system.

See end of article for authors' affiliations

Correspondence to: Miss S M Jones, Odstock Burns and Wound Healing Charitable Trust, Laing Laboratory, Salisbury District Hospital, Salisbury, Wiltshire SP2 8BJ, UK; Sophie.Jones@nhs.net

Submitted 1 July 2004 Accepted

15 September 2004
W ounds may be caused by a variety of mechanisms and can be acute or chronic. They can be progressive, disabling, and, in some cases, cause significant morbidity or mortality. All this means that management of wounds can be a complex and difficult task. In addition, time consumed and treatment costs of efforts to improve wound healing have become much greater as the population has become more demanding with higher expectations and with the increasing age of the general population.

Historically cleanliness of the wound (Pare 1545) and removal of pus (Lister 1867) ${ }^{1}$ have represented advances in the techniques of wound management and treatment. More recent important advances in wound care have occurred as a result of expansion in the knowledge of the healing process at the molecular level. This has resulted in the development of wound care methods that have greatly improved the ability of wounds to heal with fewer complications. A structured approach to wound care has developed through research and pragmatic experience. This has resulted in the development of wound care algorithms and the creation of a reconstructive ladder in surgical practice (fig 1). ${ }^{23}$ The reconstructive ladder is the schema by which increasingly complex reconstructive procedures are described. These reconstructive procedures are continually evolving to meet the specific requirements necessary to achieve satisfactory wound closure and restore functional integrity in the least time and with the least resultant morbidity. Methods include skin grafting, local skin flaps, and free tissue transfer. Each step in the reconstructive ladder describes increasingly complex procedures that require more expertise.

Wound treatment is orientated towards creating an environment in the wound that will promote healing or allow surgical intervention to cover and close the wound. The ideal intrinsic wound healing environment as proposed by Winter (1962) ${ }^{4}$ is moist (but not wet), uninfected with a good blood supply, and contains the correct balance of inflammatory mediators. However, a wound dressing or treatment modality that can accelerate the achievement of all these features in every wound is yet to be invented. Consequently, research has developed a multitude of dressings and treatment options ranging from simple gauze dressings to more complex chemical impregnated dressings. Indeed, particular dressings are now available for particular wound types (for example, moist, dry eschar, sloughy, exudative). This is often confusing and makes the choice of dressing or treatment difficult for many clinicians.

It would be impractical and expensive to stock all the different types of dressing. With the increasing numbers of complex wounds being treated within the NHS, the postulated cost for the next decade is more than $£ 2$ billion. Research is thus hunting for simple, cost effective treatment that will promote healing. Topical negative pressure (TNP) therapy is a simple treatment that is postulated to be an effective promoter of wound progress in a variety of wounds.

\section{DEVELOPMENT OF TOPICAL NEGATIVE PRESSURE}

The concept of using negative pressure to create a suction force enabling the drainage of surgical wounds to promote wound healing is well known. ${ }^{56}$ If excess fluid is not adequately removed from a wound after surgery, its components may serve as both physical and chemical deterrents to wound healing. In addition, the basic concept of mechanical forces influencing the shape and growth of tissues is well reported. A buried drain can have little mechanical affect on surrounding tissue. Thus the development of the application of suction topically across the surface of the wound to provide a solution capable of removing excess fluid and exerting a mechanical effect on the wound has been a novel concept. The technique of TNP therapy has been shown in practice to remove excess interstitial fluid and transmit a mechanical force to the

Abbreviations: TNP, topical negative pressure; PU, polyurethane; PVA, polyvinyl-alcohol; TRAC, therapeutic regulated accurate care 
surrounding tissues producing deformation of the extracellular matrix and promoting a reduction in wound size..$^{5-7}$

Argenta and Morykwas first published experimental work on TNP therapy in 1997 using animal and scientific studies. Using an acute wound model in swine, Morykwas postulated a multimodal mechanism of action. ${ }^{8}$ Since then the improved outcome parameters observed clinically in a variety of wounds have resulted in a widespread empirical uptake of TNP and acted as a catalyst for the development of a number of research programmes.

WHAT IS TOPICAL NEGATIVE PRESSURE THERAPY? The TNP therapy equipment consists of a reticulated foam dressing that is inserted in the wound and sealed in place with the use of an adhesive dressing. A suction force is then applied by a VAC (Vacuum Assisted Closure, Advanced Therapy System, KCI Whitney, Oxon, UK) machine across the wound surface (fig 2).

To achieve the application of negative pressure at the surface of the wound a polyurethane (PU) or polyvinylalcohol (PVA) foam is cut to fit the wound cavity exactly (fig 3). PU foam is black and easily deformable whereas PVA (white) foam is stiffer and requires higher pressures. Choice of foam is clinician dependent, although PU (black foam) is used more commonly in the UK. The foam is covered with an adhesive drape into which a small hole is cut and a TRAC (therapeutic regulated accurate care) pad applied over the hole. The adhesive dressing creates a sealed environment for moist healing and the TRAC pad is connected to the negative pressure generator (VAC machine) - a source for suction and drainage. Pressures achieved at the TRAC pad-foam interface are monitored by the VAC machine.

Once in position the TNP dressing provides a stable environment over the wound surface. This enables the wound to heal even in the mobile patient without shear stresses affecting the wound, which could damage the fragile newly formed tissue. It is proposed that TNP may increase patient comfort levels through removal of traditional bulky gauze dressings that can become soaked through and smell offensive. As the dressings are changed at 48 hourly intervals and the patients can be mobilised nursing input is also reduced.

Most clinicians use TNP therapy at a sub-atmospheric pressure of $125 \mathrm{~mm} \mathrm{Hg}$ using PU (black) foam-that is, $125 \mathrm{~mm} \mathrm{Hg}$ below atmospheric pressure. Evidence for this being the optimum pressure is limited. Morykwas ${ }^{9}$ showed using laser Doppler needle flow probes in the acute wound swine model that local tissue perfusion increased with increasing pressures up to $125 \mathrm{~mm} \mathrm{Hg}$. Although, further increases in pressure led to a decrease in local tissue perfusion giving a bell shaped distribution.

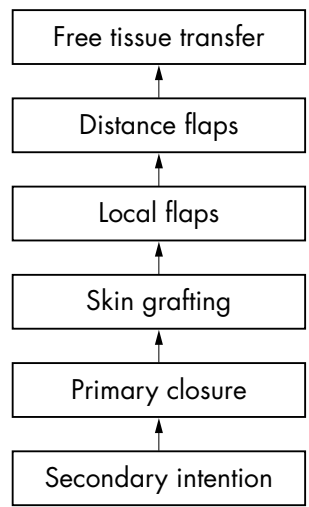

Figure 1 The reconstructive ladder.

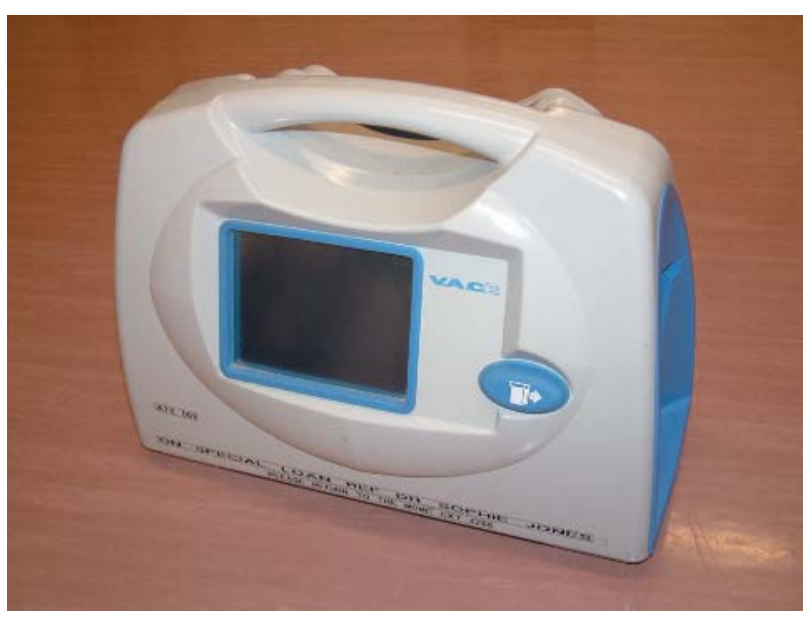

Figure 2 The VAC machine.

Morykwas and Argenta ${ }^{9}$ also showed a drop off in tissue perfusion after a period of five to seven minutes of continuous treatment. Subsequent studies undertaken to compare rates of granulation tissue formation in the healing wound under different pressure cycles of TNP therapy ${ }^{10}$ showed a faster rate of granulation tissue formation after intermittent treatment (five minutes on, two minutes off) in comparison with continuous treatment. These results however have only been shown so far in acute animal wounds and as yet have not been shown in human wounds. Nevertheless in practice many patients find the intermittent regimen difficult to tolerate because of discomfort during the changes in pressure and thus the continuous profile is more commonly used in the UK at this time.

TNP dressings should be changed every 48-56 hours except in exceptional circumstances (for example, over a skin graft). The manufacturers (KCI Limited, Ferndown, UK) recommend that dressings are also changed more frequently in the presence of (aerobic) infection. If dressings are routinely left for longer periods of time ( $>56$ hours) this can lead to increased discomfort at the time of dressing changes because of ingrowth of granulation tissue into the foam. Anecdotally the granulation tissue is also clinically found to be less healthy the longer the dressing is left in situ although there is no published literature on this. However, a number of studies were reviewed by the Cochrane report ${ }^{11}$ who felt that with the

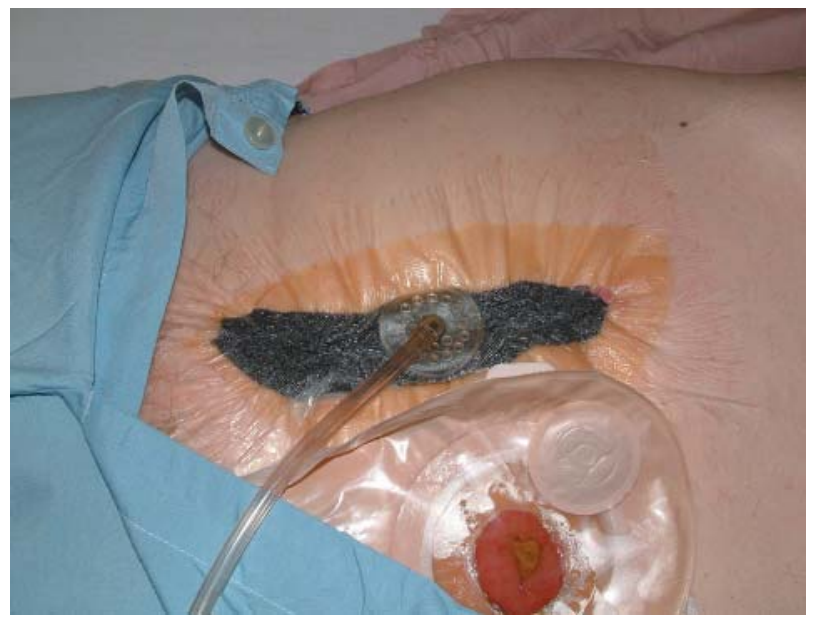

Figure 3 VAC TNP dressing in situ on an abdominal wound. 
current literature it is still not possible to determine the optimum TNP regimen.

\section{MECHANISMS OF ACTION}

Despite the early clinical success and widespread empirical introduction of TNP into clinical practice, it is not known exactly how TNP therapy may exert effects on the wound. Several mechanisms have been proposed. TNP is said to increase local blood flow and reduce oedema and bacterial colonisation rates. It is thought to promote closure of the wound by promoting the rapid formation of granulation tissue as well as by mechanical effects on the wound. It concurrently provides a moist wound environment and removes excess wound exudates thus aiding in the creation of the "ideal wound healing environment". ${ }^{4}$

\section{Local blood flow}

Morykwas ${ }^{9}$ used needle probe laser Doppler flowometry to show that sub-atmospheric pressures of $125 \mathrm{~mm} \mathrm{Hg}$ resulted in a fourfold increase in blood flow using an excisional wound model in pigs. This increase in blood flow has also been shown in human burns. ${ }^{12}$ Further higher increases in pressure (>200 $\mathrm{mm} \mathrm{Hg}$ ) were shown to decrease blood flow. There remains confusion as to whether continued pressure leads to an eventual decline in blood flow ${ }^{9}$ or a cyclical pattern of blood flow. ${ }^{13}$

These direct effects on dermal vasculature are thought to be mediated by influencing vasomotor mediators. However, the indirect effects of mechanical forces exerted on the extracellular matrix inevitably affect the microvasculature contained within it.

\section{Mechanical stress}

The importance of physical forces in TNP therapy is still theoretical, however there is good evidence of the importance of mechanical stress on cellular reproduction and angiogenesis. ${ }^{14}$ Increasing mechanical stress in vitro causes an increase in cellular activity, the nature of which varies with the cell type and methodology. Accelerated cell cycling ${ }^{15}$ and DNA synthesis $^{16}$ have been seen. Experimental evidence from model systems also suggests that mechanical forces can result in increased fibrogenesis in cutaneous wound models. ${ }^{16}$

\section{Granulation tissue formation}

In Morykwas' studies ${ }^{9}$ using porcine dorsal midline excisional full thickness excisional wound models, alginate impressions were taken daily after treatment with TNP. Volume displacement of these casts demonstrated that TNP treated wounds showed increased granulation tissue formation compared with the controls by $63 \%$ and $103.4 \%$ (continuous and intermittent suction respectively), although it is not known what effect contraction played to change the size of these wounds. This increase in granulation tissue formation has been confirmed by Joseph et al and Fabian et al using rabbit ear studies. ${ }^{17} 18$

\section{Bacterial colonisation}

Studies using swine wounds inoculated with a human isolate of Staphylococcus aureus and a swine isolate of Staphylococcus epidermidis that were treated with TNP or controlled moist saline dressing showed a more rapid decline in bacterial levels in the TNP treated wounds. ${ }^{9}$ There have been a variety of other studies confirming this. ${ }^{19-21}$

\section{Oedema reduction and exudate management}

Clinically TNP removes large amounts of fluid from wounds especially acute burns. ${ }^{22}$ The resulting reduction in oedema is thought to aid in the enhancement of blood and nutrient flow into the wound. However, this removal of exudate (which will include metalloproteinases and other inflammatory mediators $^{23}{ }^{24}$ ) from the wound and oedema from the surrounding tissues encourages nutrient movement into the wound area even if blood flow is not increased. Removal of fluid prevents a build up of inflammatory mediators and encourages diffusion of further nutrients into the wound. This is all beneficial to the healing process especially in the case of chronic wounds where it has been hypothesised that an imbalance of metalloproteinases can inhibit healing. Anecdotally the volume of wound exudate gathered from acute wounds decreases significantly over the first three to four days signifying a decrease in wound oedema. However, currently there is no quantitative evidence to support an actual reduction in interstitial wound fluid although studies are underway to attempt to evaluate changes in wound fluid constituents under TNP.

\section{OBJECTIVES OF TREATMENT}

The successful outcome of any wound treatment depends upon initial expectations. Expectations vary; in some complete healing is required, in others the objective may be the ability to send the patient home with/without dressing, or the objective may be to obtain closure by skin grafting or definitive surgery. Wounds need to be regularly inspected to ensure the wound healing is going accordingly. It is important to bear these objectives in mind with all dressings and throughout the treatment regimen with TNP therapy or any other modality. Certainly TNP therapy is not a panacea for all patients or all wounds.

\section{WOUND TYPES}

TNP has been described for use in many different types and sizes of wound. Table 1 gives many of the possible wound types. ${ }^{12}$ From this the wounds can be considered as falling into four categories: acute, chronic, as an adjunct to surgery, or for salvage procedures.

\section{Acute wounds}

Acute wounds are usually defined as wounds that heal within an expected amount of time without complication. Although the gold standard would be to close all acute wounds immediately, in many cases this is not possible whether because of patient factors or wound factors. For example, grade III or IV (Gustillo) open fractures should have soft tissue coverage within 72 hours to prevent bone infection. However, this may have to be delayed because of restricted accessibility to the required reconstructive expertise especially in cases of significant tissue loss. Thus in certain cases coverage may be postponed by the use of TNP therapy after adequate debridement of the wound. The TNP therapy will keep the wound clean until such time as surgery is feasible.

Table 1 Wounds suitable for treatment with topical negative pressure

\begin{tabular}{ll}
\hline Wound type & Examples \\
\hline Acute & Trauma (upper/lower limb) \\
& Burns \\
Chronic & Pressure sores \\
& Leg ulcers \\
& Diabetic ulcers \\
Salvage & Wound dehiscence \\
& Wound infection \\
& Necrotising fasciitis \\
& Postoperative sternal infections \\
& Skin grafts \\
Adjunct to surgery & Flap surgery \\
& Wound bed preparation \\
& \\
\hline
\end{tabular}


After complete excision of any non-viable tissues, application of TNP therapy for two to three weeks can prevent local infection, partially fill the wound with granulation tissue, and transform difficult to manage situations into more simple circumstances, for example producing exposed granulation tissue at the wound bed that can be easily covered using a skin graft. It may stimulate granulation tissue formation over bare bone. This is an important advantage in elderly or debilitated patients, in whom amputation rates can be reduced. It has also been used for degloving injuries where the degloved skin is defatted and replaced onto the wound as a skin graft under TNP. Thus in the acute scenario TNP therapy can be used either as a mechanism of downgrading the wound requirements or as an intermediary dressing before definitive treatment.

\section{Chronic wounds}

Chronic wounds are defined as wounds that do not heal within an expected time frame. In elderly patients, nutritional deficits and polypathology may preclude reconstructive surgery. Surgery in patients with neurological deficits can be difficult to treat because of the absence of neurotropic factors to protect the wound and their increased propensity to infection. The use of surgery in these wounds is thus often restricted to those cases where a clear improvement can be expected. It can be predicted that in these cases a move down the reconstructive ladder would be beneficial. TNP therapy can keep a wound clean while simultaneously stimulating granulation tissue that can reduce the reconstructive requirements of the individual wound. The usefulness of TNP has been shown in complex diabetic ulcers, chronic ulcers, and pressure sores. ${ }^{25}$

\section{Salvage procedures}

TNP is useful in the salvage of difficult cases. This dressing technique can be brought in as a "last measure" or even as a holding procedure until the patient is well enough to cope with surgery. Among other examples, salvage wounds would include dehisced abdomens and large areas of skin loss caused by necrotising fasciitis. These wounds can be large with unhealthy or infected wound beds. The patients are also often unwell and unsuitable candidates for immediate major reconstructive surgery that is reliant on a good cardiac output and peripheral blood supply. Thus in these situations TNP can be useful as a temporary wound dressing therapy that will also stimulate healing. Use of TNP therapy in these situations may lower the reconstructive requirements of the wound. ${ }^{26}$ The TNP therapy dressings also require less nursing staff input than traditional dressings as the dressings are changed less often.

\section{Adjunct to surgery}

The ideal method to maintain skin grafts over a suitable wound bed entails firm fixation, prevention of shearing forces, and adaptation to convex or concave surfaces, evacuation of sub-graft haematoma and seroma, and minimisation of infection. Splintage using TNP therapy fulfils these criteria and various studies give take rates of over $90 \%$ even in difficult contoured areas. ${ }^{27}$ TNP can also be used to prepare a wound bed for surgery and has been described as appropriate over flaps. ${ }^{28}$

\section{PROTOCOLS FOR USE}

Protocols for use of TNP therapy need to be tailored to the specific objectives for treatment for an individual patient. Thus if the purpose of the treatment is to fill a cavity wound with granulation tissue then regular dressing changes at two day intervals are undertaken until the cavity is reduced. However, if TNP is being used to hold a skin graft in place then the dressing is left in situ for five days at a lower pressure (75 mm Hg subatmospheric pressure).

TNP therapy may be used to help move the reconstructive requirements of the wound down the reconstructive ladder. This can result in a number of potential benefits for the patient and the treatment regimen. As an example a wound that would require a flap reconstruction may be reduced in size and a healthy wound bed produced that would enable application of a skin graft, a simple procedure instead of a more challenging flap reconstruction.

The manufacturers recommend that TNP dressings are routinely changed at 48 hourly intervals. If the foam dressing is adherent to the wound bed at dressing changes saline can be applied to the foam to moisten the points of adhesion, or alternatively lidocaine. In between dressing changes treatment should be active for more than 22 hours each day. Dressings should also be removed if TNP therapy is terminated as the foam and adhesive layer are not designed for exudate absorption. This would result in a macerated and engorged wound.

TNP therapy is thought to be most effective at pressures of $125 \mathrm{~mm} \mathrm{Hg}$. ${ }^{9}$ However, this has not been confirmed in clinical studies to date. Treatment should be terminated once the wounds objectives have been reached or if infection or a lack of progress is noted.

\section{CONTRAINDICATIONS}

TNP is not successful on sloughy or grossly infected wounds. The wound bed needs to be debrided using either surgical or topical desloughing agents (for example, Purilon gel). TNP therapy is also not recommended for use in bleeding wounds, malignancy, over bare vasculature or necrotic tissue. The presence of abnormal clotting, fistulas, open body cavities, or after oncological resections needs to be considered and evaluated individually and clinical judgement applied as to potential benefit applied in each case.

\section{COST IMPLICATIONS}

The daily rental charges for a VAC machine and consumables are significant. This has discouraged many from using the system. However, there have been some reports showing that the increased healing times and downgrading of required operations correlates to decreased overall costs of care. ${ }^{29}$ The dressing should also enable larger wounds to be treated in the community with minimal nursing care impact. This would free up hospital beds permitting faster throughput of operative patients and preventing waiting list build up.

\section{THE FUTURE}

TNP therapy can be regarded as a method that combines the benefit of both open and closed treatment and adheres to DeBakey's principles of being short, safe, and simple. It has been shown to work and be beneficial to wound healing, but protocols for use have to date been clinician dependent and sometimes idiosyncratic. The Cochrane report $(2001)^{11}$ stated that there is a paucity of high quality trials, and most studies currently consist of small sample sizes or methodological limitations so that any results should be interpreted with caution. Further random controlled trials are required to investigate these issues although this may be a challenging problem for researchers in wound healing because of difficulties in the assessment and measurement of healing in wounds.

TNP therapy is not the answer for all wounds; however it can make a significant difference in many cases. In much of the current literature on TNP comparing it with more traditional dressings wound healing has been measured in "time to complete healing" or epithelialisation. This may be an inappropriate measure of effectiveness as TNP is most 
useful in difficult cavity or highly exudative wounds. TNP is a useful tool in moving a wound to a point where more traditional dressings or more simple surgical reconstructive methods can be used. As such it is a well deserved, although at present pragmatic addition to the wound healing armamentarium and the reconstructive ladder.

\section{CONTRIBUTORS}

All authors have also made a substantial contribution to the production of this article.

\section{Authors' affiliations}

S M Jones, P E Banwell, P G Shakespeare, Odstock Burns and Wound Healing Charitable Trust, Laing Laboratory, Salisbury District Hospital, Salisbury, Wiltshire, UK

Funding: SJ has been in receipt of a fellowship from $\mathrm{KCl}$ Medical (Ferndown, UK) for unrelated research in topical negative pressure.

Conflicts of interest: none.

\section{REFERENCES}

1 Porter R. Surgery. Blood and guts a short history of medicine. London: Penguin, 2002:109-34.

2 Beitz JM, Bates-Jensen B. Algorithms, critical pathways, and computer software for wound care: contemporary status and future potential. Ostomy Wound Management 2001;47:33-40.

3 Levin LS. The reconstructive ladder. An orthoplastic approach. Orthop Clin North Am 1993;24:393-409.

4 Winter GD. Formation of the scab and the rate of epithelization of superficial wounds in the skin of the young domestic pig. Nature 1962;193:293-4.

5 Fox JW, Golden GT. The use of drains in subcutaneous surgical procedures. Am J Surg 1976;132:673-4.

6 Fay MF. Drainage systems. Their role in wound healing. AORN J 1987:46:442-55.

7 Urschel JD, Scott PG, Williams HT. The effect of mechanical stress on soft and hard tissue repair; a review. Br J Plast Surg 1988;41:182-6.

8 Argenta LC, Morykwas MJ. Vacuum-assisted closure: a new method for wound control and treatment: clinical experience. Ann Plast Surg 1997:38:563-76

9 Morykwas MJ, Argenta LC, Shelton-Brown El, et al. Vacuum-assisted closure: a new method for wound control and treatment: animal studies and basic foundation. Ann Plast Surg 1997;38:553-62.

10 Morykwas MJ, Faler BJ, Pearce DJ, et al. Effects of varying levels of subatmospheric pressure on the rate of granulation tissue formation in experimental wounds in swine. Ann Plast Surg 2001;47:547-51.
11 Evans D, Land L. Topical negative pressure for treating chronic wounds. Cochrane Library. Issue 1. Oxford: Update Software, 2001.

12 Banwell PE, Teot L. Topical negative pressure (TNP): the evolution of a novel wound therapy. J Wound Care 2003;12:22-8.

13 Banwell PE, Jones SM, Evison D. Topical negative pressure modulates dermal microvascular blood flow dynamics and temperature profiles at the wounddressing interface. J Wound Care (in press).

14 Ichioka S, Shibata M, Kosaki K, et al. Effects of shear stress on wound-healing angiogenesis in the rabbit ear chamber. J Surg Res 1997:72:29-35.

15 Curtis AS, Seehar GM. The control of cell division by tension or diffusion. Nature 1978;274:52-3.

16 Brunette DM. Mechanical stretching increases the number of epithelial cells synthesizing DNA in culture. J Cell Sci 1984;69:35-45.

17 Fabian TS, Kaufman HJ, Lett ED, et al. The evaluation of subatmospheric pressure and hyperbaric oxygen in ischemic full-thickness wound healing. Am Surg 2000;66:1136-43

18 Joseph E, Hamori CA, Bergman S, et al. A prospective randomised trial of vaccum-assisted closure versus standard therapy of chronic nonhealing wounds. Wounds 2000:3:60-7.

19 Fleischmann W, Becker U, Bischoff $M$, et al. Vacuum sealing: indication, technique and results. European Journal of Orthopaedic Surgery and Traumatology 1995:5:37-40.

20 Mullner T, Mrkonjic L, Kwasny O, et al. The use of negative pressure to promote the healing of tissue defects: a clinical trial using the vacuum sealing technique. Br J Plast Surg 1997;50:194-9.

21 Giovanni UM, Demaria RG, Otman S, et al. Treament of poststernotomy wounds with negative pressure. Plast Reconstr Surg 2002;109:1747.

22 Morykwas MJ, David LR, Schneider AM, et al. Use of subatmospheric pressure to prevent progression of partial-thickness burns in a swine model. J Burn Care Rehabil 1999;20:15-21

23 Buttenschoen K, Fleischmann W, Haupt $U$, et al. The influence of vacuum assisted closure on inflammatory tissue reactions in the postoperative course of ankle fractures. Foot and Ankle Surgery 2001;7:165-73.

24 Gustafsson R, Johnsson P, Algotsson L, et al. Vacuum-assisted closure therapy guided by $C$-reactive protein level in patients with deep sternal wound infection. J Thorac Cardiovasc Surg 2002;123:895-900.

25 Sibbald RG, Mahoney J. A consensus report on the use of vacuum-assisted closure in chronic, difficult-to-heal wounds. Ostomy Wound Manage 2003;49:52-66.

26 Garner GB, Ware DN, Cocanour CS, et al. Vacuum-assisted wound closure provides early fascial reapproximation in trauma patients with open abdomens. Am J Surg 2001;182:630-8.

27 Schneider AM, Morykwas MJ, Argenta LC. A new and reliable method of securing skin grafts to the difficult recipient bed. Plast Reconstr Surg 1998;102:1195-8.

28 Greer SE, Longaker MT, Margiotta $M$, et al. The use of subatmospheric pressure dressing for the coverage of radial forearm free flap donor-site exposed tendon complications. Ann Plast Surg 1999;43:551-4.

29 Philbeck TE Jr, Whittington KT, Millsap MH, et al. The clinical and cost effectiveness of externally applied negative pressure wound therapy in the treatment of wounds in home healthcare Medicare patients. Ostomy Wound Manage 1999;45:41-50. 